Co., Pfizer Japan Inc., Daiichi Sankyo Co., Ltd., Shionogi \& Co., Ltd., Sanofi K.K., Nippon Kayaku Co., Ltd., Yutoku Pharmaceutical Ind. Co., Ltd., UCB Japan Co. Ltd., Nihon Pharmaceutical Co., Ltd., and Bayer Yakuhin, Ltd., Consultant of: UCB Japan Co., Ltd., Eisai Co., Ltd., and Chugai Pharmaceutical Co., Speakers bureau: Mitsubishi-Tanabe Pharma Co., Chugai Pharmaceutical Co., Eisai Co., Ltd., Astellas Pharma Inc., Janssen Pharmaceutical K.K., Ayumi Pharmaceutical Co., Pfizer Japan Inc., Asahikasei Pharma Corp., Sanofi K.K., Novartis Pharma K.K., Eli Lilly Japan K.K., Nippon Kayaku Co., Ltd., Teijin Pharma Ltd., Takeda Pharmaceutical Co., Nippon Boehringer Ingelheim Co., Ltd., and AbbVie GK. DOI: 10.1136/annrheumdis-2020-eular.2531

\section{FRI0369 MIMICKING GLUCOCORTICOID-INDUCED OSTEOPOROSIS USING AN IN VITRO TRABECULAR HUMAN BONE MODEL}

A. Lang ${ }^{1,2}$, K. Diesing ${ }^{1}$, A. Damerau ${ }^{1,2}$, S. Uzun ${ }^{1}$, M. Pfeiffenberger ${ }^{1,2}$ T. Gaber ${ }^{1,2}$, F. Buttgereit ${ }^{1,2} .{ }^{1}$ Charité-Universitätsmedizin Berlin, Department for Rheumatology and Clinical Immunology, Berlin, Germany; ${ }^{2}$ German Rheumatism Research Center, Berlin, Germany

Background: The bone matrix consists of inorganic and organic components and a variety of specialized cells such as osteoblasts, osteocytes and osteoclasts. The bone-forming osteoblasts are responsible for the production of organic matrix components; they differentiate later into osteocytes which is accompanied by matrix mineralization. Osteoclasts are multinuclear giant cells, which resorb bone. Healthy bone homeostasis is characterized by a balanced, dynamic and continuous remodeling process. Glucocorticoids (GCs) are commonly used to successfully treat patients with inflammatory rheumatic and other autoimmune diseases. However, long-term treatment with GC can potentially lead to several adverse effects such as the inhibition of osteoblast proliferation and the increase of osteoclastic activity resulting in osteoporosis.

Objectives: Hence, the aim of our project is to i) develop an in vitro trabecular human bone model, ii) integrate this bone model into a perfusion system to accelerate mineralization and provide biomechanical stimuli and iii) applying prednisolone to induce osteoporosis. Here we present our initial results describing the successful differentiation of osteoblasts and osteoclasts in a 3D environment, and the accomplished integration of the bone model into a perfusion system.

Methods: In a first step, different cultivation conditions were tested to allow optimal osteogenic or osteoclastic differentiation. To this end, a) human bone marrow derived mesenchymal stromal cells (hMSCs) were treated with osteogenic medium, and b) monocytes (isolated from buffy coats) were differentiated into osteoclasts using following protocol: incubation for 3 days with $25 \mathrm{ng} / \mathrm{ml} \mathrm{M-CSF}$ followed by an 18-day incubation with M-CSF and $50 \mathrm{ng} / \mathrm{ml}$ RANKL. Calcification of hMSCs was evaluated via Alizarin Red S staining. Osteoclasts were identified using immunofluorescence staining observing multinucleated (DAPI) giant (B-Actin) cells with TRAP and Cathepsin $\mathrm{K}$ activity. Additional gene expression analyses are currently conducted using qRT-PCR and looking for osteoclastspecific genes. In parallel to the monolayer cultures, cells were transferred on $\beta$-tricalcium phosphate ( $\beta T C P)$ - a suitable bony-like scaffold. Furthermore, first experiments in a dynamic bioreactor platform (OSPIN GmH) were conducted to evaluate the influence of shear stress on the cells and model systems.

Results: We have been able to populate the $\beta$ TCP scaffold with monocytes, which were differentiated into osteoclasts (morphological changes) without any effect on cellular viability as measured by Live/Dead staining. The morphological changes of those osteoclasts such as formation of filopodia could be demonstrated by scanning electron microscopy. In addition, the cultivation of $\beta$ TCP populated with hMSCs in a perfusion system showed the upregulation of osteogenic markers (RUNX2, OSX) on mRNA-level.

Conclusion: These first results of our approach to develop an in vitro 3D model for glucocorticoid-induced osteoporosis are promising. Our next step will be the co-cultivation of osteoblasts and osteoclasts under dynamic and optimized cultivation conditions. By combining several cell types, a suitable scaffold and biomechanical stimuli (perfusion), we aim to provide a valid testing platform to study underlying disease mechanisms and for drug development.

Acknowledgments: The project has been funded by the Elsbeth Bonhoff Foundation.

Disclosure of Interests: Annemarie Lang: None declared, Karoline Diesing: None declared, Alexandra Damerau: None declared, Sümeyye Uzun: None declared, Moritz Pfeiffenberger: None declared, Timo Gaber: None declared, Frank Buttgereit Grant/research support from: Amgen, BMS, Celgene, Generic Assays, GSK, Hexal, Horizon, Lilly, medac, Mundipharma, Novartis, Pfizer, Roche, and Sanofi.

DOI: 10.1136/annrheumdis-2020-eular.2569

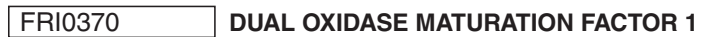 POSITIVELY REGULATES RANKL-INDUCED OSTEOCLASTOGENESIS VIA ACTIVATING REACTIVE OXYGEN SPECIES PRODUCTION AND TRAF6- MEDIATED SIGNALING}

C. H. Lee ${ }^{1}$, C. H. Chung ${ }^{1}$, Y. J. Choi ${ }^{2}$, W. H. Yoo ${ }^{2}$, J.Y. Kim ${ }^{3}$, M. S. Lee ${ }^{1}$. ${ }^{1}$ Wonkwang University Hospital, Internal Medicine, Iksan, Korea, Rep. of (South Korea); ${ }^{2}$ Chonbuk National University Hospital, Internal Medicine, Jeonju, Korea, Rep. of (South Korea); ${ }^{3}$ Wonkwang University, School of Medicine, Iksan, Korea, Rep. of (South Korea)

Background: Reactive oxygen species (ROS) are one of the significant factors of chemical or physical cell signaling in a wide variety of cell types including skeletal cells. Receptor activator of NF- $\beta B$ ligand (RANKL) induces generation of intracellular ROS, which act as second messengers in RANKL-mediated osteoclastogenesis. Dual oxidase maturation factor 1 (Duoxa1) was first identified as a Drosophila Numb-interacting protein (NIP), and has been associated with the maturation of ROS generating enzymes including dual oxidases (Duox1 and Duox2). In the progression of osteoclast differentiation using mouse bone marrow-derived macrophages (BMMs), we identified that only Duoxa1 level showed an effective change upon RANKL stimulation, but not Duox1, Duox2, and Duoxa2.

Objectives: we hypothesized that Duoxa1 could independently act as a second messenger for RANKL stimulation and regulate ROS production during osteoclast differentiation.

Methods: Using siRNA or retrovirus transduction and knockdown of Duoxa1 via SiRNA

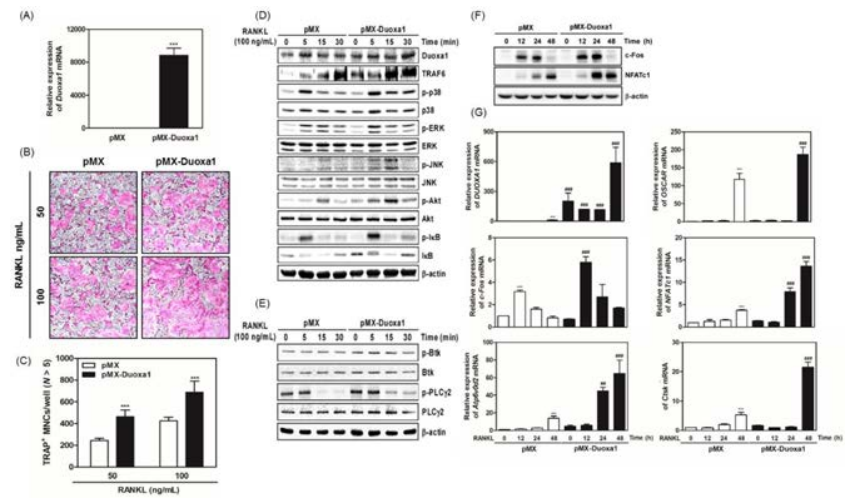

Results: Duoxa1 level gradually increased during RANKL-induced osteoclast differentiation. We found that Duoxa1 regulated RANKL-stimulated osteoclast formation and bone resorption positively. knockdown of Duoxa1 via siRNA decreased the RANKL-induced ROS production. During Duoxa1-related control of osteoclastogenesis, activation of tumor necrosis factor receptor (TNFR)-associated factor 6 (TRAF6)-mediated early signaling molecules including MAPKs, Akt, I $\beta B$, Btk, and PLC 2 was affected, which sequentially modified the mRNA or protein expression levels of key transcription factors in osteoclastogenesis, such as c-Fos and NFATc1, as well as mRNA expression of osteoclast-specific markers including OSCAR, ATP6v0d2, and CtsK.

Conclusion: Overall, our data indicate that Duoxa1 plays a crucial role in osteoclastogenesis via regulating RANKL-induced intracellular ROS production and activating TRAF6-mediated signaling.

Disclosure of Interests: None declared

DOI: 10.1136/annrheumdis-2020-eular.3505

\begin{tabular}{|l|l|}
\hline FRI0371 & BETULINIC ACID INHIBITS RANKL-INDUCED \\
OSTEOCLASTOGENESIS VIA ATTENUATING \\
AKT, NF-KB, AND PLCG2-CA2+ SIGNALING AND \\
PREVENTS INFLAMMATORY BONE LOSS
\end{tabular}

C. H. Lee ${ }^{1}$, C. H. Chung ${ }^{1}$, J. Y. Kim², Y. J. Choi ${ }^{3}$, W. H. Yoo ${ }^{3}$, M. S. Lee ${ }^{1}$ ${ }^{1}$ Wonkwang University Hospital, Internal Medicine, Iksan, Korea, Rep. of (South Korea); ${ }^{2}$ Wonkwang University, School of Medicine, Iksan, Korea, Rep. of (South Korea): ${ }^{3}$ Chonbuk National University Hospital, Internal Medicine, Jeonju, Korea, Rep. of (South Korea)

Background: Betulinic acid (BA), a natural plant-derived pentacyclic triterpenoid compound, is known to possess numerous pharmacological and biochemical properties including anti-inflammatory, anti-cancer, and anti-adipogenic activity. 